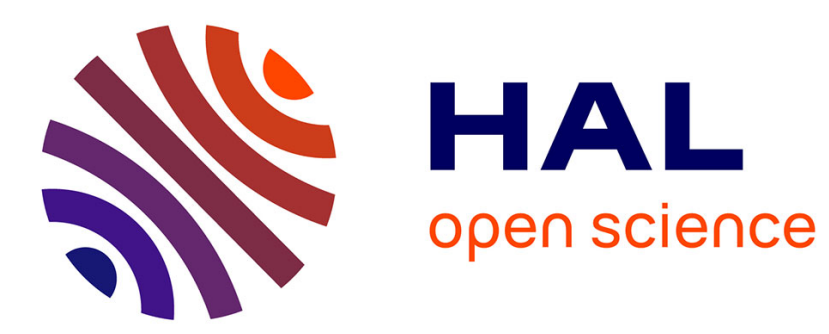

\title{
Impact of transposable elements on genome size variation between two closely related crustacean species
}

Thomas Becking, Clement Gilbert, Richard Cordaux

\section{To cite this version:}

Thomas Becking, Clement Gilbert, Richard Cordaux. Impact of transposable elements on genome size variation between two closely related crustacean species. Analytical Biochemistry, 2020, 10.1016/j.ab.2020.113770 . hal-03020081

\section{HAL Id: hal-03020081 \\ https://hal.science/hal-03020081}

Submitted on 1 Dec 2020

HAL is a multi-disciplinary open access archive for the deposit and dissemination of scientific research documents, whether they are published or not. The documents may come from teaching and research institutions in France or abroad, or from public or private research centers.
L'archive ouverte pluridisciplinaire HAL, est destinée au dépôt et à la diffusion de documents scientifiques de niveau recherche, publiés ou non, émanant des établissements d'enseignement et de recherche français ou étrangers, des laboratoires publics ou privés. 


\section{Impact of transposable elements on genome size variation between \\ 2 two closely related crustacean species}

4 Thomas Becking ${ }^{\mathrm{a}}$, Clément Gilbert ${ }^{\mathrm{a}, \mathrm{b}}$ and Richard Cordaux ${ }^{\mathrm{a},{ }^{*}}$

5

6 a Laboratoire Ecologie et Biologie des Interactions, UMR CNRS 7267, Université de Poitiers,

7 Poitiers, France

8 b Present address: Laboratoire Evolution, Génomes, Comportement, Écologie, Unité Mixte de 9 Recherche 9191 Centre National de la Recherche Scientifique and Unité Mixte de Recherche 10247 Institut de Recherche pour le Développement, Université Paris-Sud, 91198 Gif-sur11 Yvette, France.

$13{ }^{*}$ Corresponding author: richard.cordaux@univ-poitiers.fr 


\section{Abstract}

Identifying and quantifying genome size variation among species and understanding if it evolves as a consequence of adaptive or stochastic processes is still a long-standing objective in evolutionary biology. Here, we investigated the basis of genome size variation between two closely related species of terrestrial isopods: Armadillidium vulgare and Armadillidium nasatum (Crustacea, Oniscidea). The two species diverged 25 million years ago and they substantially diverge in genome size, the $A$. vulgare genome being $~ 500$ megabases larger than the A. nasatum genome (1.7 vs. 1.2 gigabases, respectively). Our analyses indicated that genome size difference is essentially attributed to transposable elements (TEs). As the two genomes largely share the same TE families, differential transpositional activity likely underlies the observed variation in TE copy numbers and genome size. Analyses of TE expression suggested that there may be $\sim 4$ times more transcribed TE copies in $A$. vulgare than in A. nasatum. However, the cumulative expression level of all expressed TEs was higher in A. nasatum than in A. vulgare, suggesting that the two species may have recently been experiencing different TE transposition dynamics. Overall, our results illustrate the important impact TEs can have on genome structure and evolution between closely related species.

\section{Keywords}

transposable elements, genome sequencing, comparative analysis, crustaceans, terrestrial isopods, genome size 


\section{Introduction}

Genome size variation is one of the most noteworthy sources of biodiversity on Earth [1]. The nature and importance of the evolutionary forces and the molecular mechanisms causing this variation are the subject of numerous studies and debates [2-8]. Many studies have shown that genome size is positively correlated to the content in transposable elements (TEs), as for example in mammals [9], birds [9], insects [10-16], or flowering plants $[17,18]$. However, the reasons explaining why TEs accumulate more in some species than in others remain largely unknown.

To date, more than 4,000 genomes of Eukaryota have been sequenced and are available in GenBank (last accessed on December 2019). Crustaceans, however, are poorly represented among these genomes. Despite the existence of more than 70,000 living species of crustaceans [19], the genomes of only 30 species are available to date. While crustaceans are primarily marine organisms, constituting a large proportion of ocean biomass [20], many species have colonized freshwater, semi-terrestrial or terrestrial environments. Recently, the genomes of two species of terrestrial isopods have been assembled: Armadillidium vulgare [21] and Armadillidium nasatum [22]. These two species are closely related and they diverged from a common ancestor 25 million years ago [23]. However, it turns out that the genome assembly of $A$. vulgare (1.7 Gb) [21] is $\sim 500$ megabases larger than that of $A$. nasatum (1.2 Gb) [22]. In both cases, the genomes were sequenced using the same hybrid strategy combining Illumina short reads and PacBio long reads, and following the same assembly protocol $[21,22]$. These resources offer an interesting opportunity to compare genome size evolution between two closely related species. In this study, we used a comparative approach to investigate the causes of the large genome size variation observed between the two closely related species of terrestrial isopods $A$. vulgare and $A$. nasatum. Our results indicate that differential transpositional activity of the same TE families likely underlies most of the variation in genome size between the two species. 


\subsection{Genomic data}

The genomes of $A$. vulgare (accession number : SAUD00000000) and A. nasatum (accession number : SEYY00000000) were both assembled using a hybrid approach combining short paired-end Illumina reads and long PacBio reads and the exact same suite of software, as described previously [21] [22]. The A. vulgare assembly is composed of 43,541 contigs/scaffolds, totaling $1.725 \mathrm{~Gb}$ with an $\mathrm{N}_{50}$ (minimum contig length to cover $50 \%$ of the genome) of $51,088 \mathrm{bp}$. Based on a BUSCO analysis, we found that it includes $92.0 \%$ of the 1,066 specific arthropod core genes [24]. The A. nasatum assembly is composed of 25,196 contigs/scaffolds, totaling $1.223 \mathrm{~Gb}$ with an $\mathrm{N}_{50}$ of $86,284 \mathrm{bp}$ and including $93.9 \%$ of the 1,066 specific arthropod core genes.

\subsection{Comparison of orthologous genes}

To identify orthologous genes between $A$. vulgare and $A$. nasatum genome assemblies, protein sequences were obtained for the two species based on the structural and functional annotation of both assemblies and they were compared using the ortholog research tool OrthoMCL [25]. In total, 19,051 and 14,636 proteins were used for $A$. vulgare and $A$. nasatum, respectively. The BLASTP E-value cutoff of the OrthoMCL pipeline was set to $10^{-10}$.

\subsection{Analyses of TE expression}

To identify the genomic origin of expressed TEs, we used the pipeline TEcandidates [26]. In brief, TEcandidates uses de novo transcriptome assembly to assess expressed TE instances. To restrict reads to repeat zones, TEcandidates first runs a pre-alignment of the reads on the genome and keeps the reads that map to RepeatMasker predictions. Then, a de novo transcriptome assembly is performed using Trinity v2.4.0 with default parameters [27]. Generated contigs are mapped to the reference genome, and their intersection with 
annotated TEs is assessed with BEDtools v2.26 [28]. We used the multifasta files corresponding to $A$. vulgare and $A$. nasatum genomes, the annotation gff files generated by RepeatMasker (as each genome annotation has resolution at the level of TE instance), and RNAseq datasets generated previously (100 bp-long single end reads) [29]: 13 datasets for $A$. vulgare (SRA accession number : SRX564995 to SRX565004) and two for A. nasatum (SRX564993 and SRX564994). TEcandidates pipeline was then used with default parameters, as recommended by the authors instructions. RNAseq read quality was analyzed with FastQC (version 0.11.4, http://www.bioinformatics.babraham.ac.uk/projects/89fastqc). Removal of low-quality reads and sequencing adaptors was performed with Trimmomatic (version 0.33), setting the ILLUMINACLIP palindrome clip threshold at 30 and the simple clip threshold at 10. The quality threshold was set to a minimum Phred score of 20.

To quantify TE expression, we mapped the RNAseq reads onto the potentially expressed TEs identified by TEcandidates. Reads from multiple RNAseq datasets were mapped independently using Bowtie2 [30] using the very sensitive mode (parameter : endto-end). TE genomic coverage was calculated using BEDTools [28], and the Artemis software (v18.0.0) [31] was used to quantify expressed transcripts, and yielded reads per kilobase of exon model per million mapped (RPKM) expression values.

\section{Results \& Discussion}

\subsection{Gene content}

To investigate the possible factors underlying the difference in genome size between A. vulgare and $A$. nasatum, we first compared the gene content of the two species. The structural annotation identified 19,051 and 14,636 genes in A. vulgare and A. nasatum, respectively. The large difference in gene number between the two genomes $(4,415)$ corresponds to $26.6 \mathrm{Mb}$, as all genes combined represent $164.5 \mathrm{Mb}$ in A. vulgare [21] and 137.9 $\mathrm{Mb}$ in $A$. nasatum [22]. Thus, variation in gene number only accounts for $5 \%$ of the genome size difference between the two species. 
To investigate the possible causes underlying the difference in gene number between

the two species, all proteins annotated from the genomes of $A$. vulgare and $A$. nasatum were classified by OrthoMCL in 9,140 groups of orthologous proteins (orthogroups). Among them, 8,036 orthogroups contained sequences belonging to both $A$. vulgare and $A$. nasatum, for a total of 10,815 and 9,429 proteins (Figure 1, Table 1). The OrthoMCL analysis further identified 8,236 and 5,207 genes specific to $A$. vulgare and $A$. nasatum, respectively (Figure 1), of which 6,390 and 4,226, respectively, are present as single-copy genes (Table 1).

As both species appear to exhibit high numbers of specific genes, it is possible that some of them are artifacts of the annotation process. Indeed, a higher proportion of genes with unknown function is found among species-specific genes relative to genes common to both species (Table 1). It is conceivable that some species-specific genes may in fact be TE genes that would have been misidentified and mistakenly attributed to non-TE genes (without known function), and this may have affected $A$. vulgare to a larger extent than $A$. nasatum because both species differ in their TE content (see section 3.2 below). In addition, our results suggest that after the divergence between the two terrestrial isopods, the genome of $A$. vulgare has undergone more gene duplications than that of $A$. nasatum (Figure 1). Gene duplication is a major force behind biological innovation and is often associated with speciation phenomena [32]. This innovative force has been characterized many times in the plant kingdom [33-36] as well as in animals $[37,38]$. Thus, the difference in gene number might have contributed to the divergence between $A$. vulgare and $A$. nasatum. As it has the potential to increase phenotypic plasticity [39], gene duplication may confer a better adaptive potential, particularly during the colonization of new environments, as recently demonstrated in aphids [40]. However, it is unclear if such a hypothesis may hold for $A$. vulgare relative to $A$. nasatum, because both species are known to share the same distribution areas, and they are therefore potentially subject to similar environmental constraints [41]. In sum, while further investigations are needed to better understand the evolutionary basis of gene number variation in the two terrestrial isopod species, this parameter does not explain the difference in genome size between $A$. vulgare and $A$. nasatum. 

similarly high amounts: $303.6 \mathrm{Mb}$ (or $17.6 \%$ of the genome) for $A$. vulgare and $285.4 \mathrm{Mb}$ (or $23.3 \%$ of the genome) for $A$. nasatum (Figure 2, Table 2). Thus, tandem repeats do not contribute much to the difference in genome size between $A$. vulgare and $A$. nasatum. In fact, the difference in size between the two genomes is mainly explained by a much larger number of TE copies ( 0.6 million) in $A$. vulgare relative to $A$. nasatum. Indeed, there are $\sim 2.1$ million TE copies (or fragments) annotated by RepeatMasker in A. vulgare vs. only 1.5 million in $A$. nasatum (Table 2). Consequently, the genome of $A$. vulgare has $854.1 \mathrm{Mb}$ derived from TEs while the genome of $A$. nasatum has only 484.7 Mb (Figure 2, Figure 3, Table 2). Thus, $74 \%$ of the observed size difference between the two genomes is accounted for by TEs (Table 2).

Interestingly, the activity peak of TEs in A. nasatum corresponds to copies of TEs with $\sim 10 \%$ divergence from their consensus sequence, while the activity peak of TEs in A. vulgare corresponds to copies of TEs with $6 \%$ divergence from their consensus sequence (Figure 3). The age profile thus suggests that $A$. vulgare underwent a peak in transpositional activity at about the time of or not long after the divergence between $A$. vulgare and $A$. nasatum (estimated to $\sim 25$ million years ago or $\sim 6 \%$ divergence [23]). This is likely to explain the difference in TE abundance between the two genomes. The alternative explanation is that $A$. nasatum has a higher deletion rate compared to $A$. vulgare. Unfortunately, no outgroup is available for testing this hypothesis. In the absence of mechanisms known to specifically delete TEs, the most plausible explanation for the observed genome size differences currently remains that of a higher transpositional activity in $A$. vulgare than in A. nasatum. According to our analyses, the most represented TEs in both species belong to the LINE category (accounting for $>20 \%$ of each genome, Figure 2 ). These LINEs are divided into 14 families shared by both species (Figure 3). The second most represented TEs are DNA transposons, which represent $16.2 \%$ of the $A$. vulgare genome and $12.8 \%$ of the $A$. nasatum genome (Figure 2). These DNA transposons belong to 17 families shared by both genomes (Figure 3). The third most represented TEs are LTR retrotransposons which represent $5-8 \%$ of 
both genomes (Figure 2), distributed in 10 families shared by both species (Figure 3). In sum, the difference in TE numbers between $A$. vulgare and $A$. nasatum is not due to the acquisition and expansion of new TE families in $A$. vulgare relative to A. nasatum. Instead, differential amplification of the same TE families appears to underly variation in TE content between the two species.

In line with a growing body of literature derived from diverse taxonomic groups ranging from vertebrates $[9,42,43]$ to invertebrates $[11-14,44]$ and plants [45-47], our analysis shows that genome size variation across two closely related crustacean species can largely be explained by differences in TE content. As the divergence time between A. vulgare and $A$. nasatum is estimated to $\sim 25$ million years and genome size difference due to TE abundance is roughly $370 \mathrm{Mb}$ (Table 2), genome size divergence between $A$. vulgare and $A$. nasatum is estimated to $\sim 15 \mathrm{Mb}$ per million years. Considering genome sizes in the gigabase range, this rate may not seem stunning, but it appears to be strong enough to generate substantial genome size differences at an evolutionary time scale.

\subsection{Comparison of TE expression profiles in A. vulgare and A. nasatum}

As the larger genome size of $A$. vulgare is likely explained by a higher rate of TE amplification since the last common ancestor it shares with $A$. nasatum, we assessed whether this higher amplification rate was associated with higher levels of TE expression. The TEcandidate pipeline identified 5,317 TE copies (distributed in 4,055 different contigs) potentially transcribed in $A$. vulgare $(0.25 \%$ of the total number of TE copies) compared to 1,259 in A. nasatum ( $0.08 \%$ of the total number of TE copies, distributed in 1,101 genomic contigs) (Table 3 ). This analysis suggested that there may be $\sim 4$ times more transcribed TE copies in A. vulgare than in A. nasatum. In both species, LINEs were the TEs with the highest number of transcribed copies TEs, comprising $>50 \%$ of all transcribed copies, whereas DNA transposons accounted for $\sim 25 \%$ of all transcribed copies (Table 3).

To quantify TE expression levels in the two genomes, we carried out RPKM analyses based on RNAseq data. It appears that the most highly expressed TEs in A. vulgare and $A$. 
nasatum are different. Indeed, CR1-Zenon LINEs are by far the most highly expressed TEs in A. vulgare, followed by unclassified DNA elements, unclassified LINEs, RTE-BovB LINEs and Gypsy LTR retrotransposons (Figure 4). In contrast, unclassified DNA transposons are by far the most highly expressed TEs in A. nasatum, followed by CR1-Zenon LINEs, Gypsy LTR retrotransposons RTE-BovB LINEs and unclassified LINEs (Figure 4). Interestingly, the cumulative expression level of all expressed TEs was higher in A. nasatum than in A. vulgare, with total cumulative RPKM values of 778,709 and 686,967 , respectively. Thus, our results suggest that the species with the lowest, not the highest, TE amplification rate is associated with the highest TE expression level. Assuming that TE expression level is a good proxy for TE transpositional activity, our results may reflect that TE transposition rate is varying in opposite directions in the two species: it may be declining in $A$. vulgare after a phase of intense amplification while it may be rising or steady in A. nasatum. In any event, identification of cases of TE horizontal transfer during the recent evolutionary history of $A$. vulgare [48] supports recent transpositional activity in this species, although further investigations will be needed to evaluate its magnitude.

\section{Conclusion}

In summary, our analyses demonstrated that even though the $A$. vulgare genome contains almost 4,500 more annotated genes than that of $A$. nasatum, this difference does not contribute much to the observed difference in the genome size of $A$. vulgare relative to A. nasatum. Instead, the large difference in genome size ( $~ 500$ megabases) between the two closely related terrestrial isopods $A$. vulgare and $A$. nasatum can essentially be explained by a difference in TE content likely caused by a higher transposition activity in A. vulgare than in $A$. nasatum, subsequent to the divergence between the two species $\sim 25$ million years ago. Differential transpositional activity of the same TE families apparently underlies the variation in TE copy numbers and genome size between the two species. Our results highlight the tremendous impact of TEs on the structure and evolution of genomes previously observed in a broad range of organisms, and extended here to two closely related crustacean species. 
245 Acknowledgments

246 This work was funded by Agence Nationale de la Recherche Grant ANR-15-CE32-0006-01

247 (CytoSexDet) to R.C.

248

249 Authors Contributions:

250 Conceptualization: T.B, C.G. and R.C.; Funding acquisition: R.C; Methodology: T.B, C.G. and

251 R.C; Supervision: C.G. and R.C.; Visualization: T.B; Writing - Writing - Original Draft

252 Preparation: T.B, C.G. and R.C.; Writing - Review \& Editing: T.B, C.G. and R.C.

253

254 Conflicts of Interest:

255 The authors declare no conflict of interest.

256 
[1] T.R. Gregory, J.A. Nicol, H. Tamm, B. Kullman, K. Kullman, I.J. Leitch, B.G. Murray, D.F. Kapraun, J. Greilhuber, M.D. Bennett, Eukaryotic genome size databases, Nucleic Acids Res. 35 (2007) D332-D338. https://doi.org/10.1093/nar/gkl828.

[2] D.A. Petrov, Evolution of genome size: new approaches to an old problem, Trends Genet. 17 (2001) 23-28. https://doi.org/10.1016/S0168-9525(00)02157-0.

[3] T.R. Gregory, The Evolution of the Genome, Academic Press, Burlington, MA, 2005.

[4] M. Lynch, The Origins of Genome Architecture, 1st ed., OUP USA, Sunderland, Mass, 2007.

[5] S. Linquist, K. Cottenie, T.A. Elliott, B. Saylor, S.C. Kremer, T.R. Gregory, Applying ecological models to communities of genetic elements: the case of neutral theory, Mol. Ecol. 24 (2015) 3232-3242. https://doi.org/10.1111/mec.13219.

[6] A. Canapa, M. Barucca, M.A. Biscotti, M. Forconi, E. Olmo, Transposons, Genome Size, and Evolutionary Insights in Animals, Cytogenet. Genome Res. 147 (2015) 217-239. https://doi.org/10.1159/000444429.

[7] T.A. Elliott, T.R. Gregory, What's in a genome? The C-value enigma and the evolution of eukaryotic genome content, Philos. Trans. R. Soc. B Biol. Sci. 370 (2015) 20140331. https://doi.org/10.1098/rstb.2014.0331.

[8] T.A. Elliott, T.R. Gregory, Do larger genomes contain more diverse transposable elements?, BMC Evol. Biol. 15 (2015) 69. https://doi.org/10.1186/s12862-015-0339-8.

[9] A. Kapusta, A. Suh, C. Feschotte, Dynamics of genome size evolution in birds and mammals, Proc. Natl. Acad. Sci. 114 (2017) E1460-E1469. https://doi.org/10.1073/pnas.1616702114.

[10] D.A. Petrov, E.R. Lozovskaya, D.L. Hartl, High intrinsic rate of DNA loss in Drosophila, Nature. 384 (1996) 346-349. https://doi.org/10.1038/384346a0.

[11] X. Wang, X. Fang, P. Yang, X. Jiang, F. Jiang, D. Zhao, B. Li, F. Cui, J. Wei, C. Ma, Y. Wang, J. He, Y. Luo, Z. Wang, X. Guo, W. Guo, X. Wang, Y. Zhang, M. Yang, S. Hao, B. Chen, Z. Ma, D. Yu, Z. Xiong, Y. Zhu, D. Fan, L. Han, B. Wang, Y. Chen, J. Wang, L. Yang, W. Zhao, Y. Feng, G. Chen, J. Lian, Q. Li, Z. Huang, X. Yao, N. Lv, G. Zhang, Y. Li, J. Wang, J. Wang, B. Zhu, L. Kang, The locust genome provides insight into swarm formation and longdistance flight, Nat. Commun. 5 (2014) 1-9. https://doi.org/10.1038/ncomms3957.

[12] C. Sessegolo, N. Burlet, A. Haudry, Strong phylogenetic inertia on genome size and transposable element content among 26 species of flies, Biol. Lett. 12 (2016) 20160407. https://doi.org/10.1098/rsbl.2016.0407.

[13] V. Talla, A. Suh, F. Kalsoom, V. Dincă, R. Vila, M. Friberg, C. Wiklund, N. Backström, Rapid Increase in Genome Size as a Consequence of Transposable Element Hyperactivity in Wood-White (Leptidea) Butterflies, Genome Biol. Evol. 9 (2017) 24912505. https://doi.org/10.1093/gbe/evx163.

[14] S.S. Lower, J.S. Johnston, K.F. Stanger-Hall, C.E. Hjelmen, S.J. Hanrahan, K. Korunes, D. Hall, Genome Size in North American Fireflies: Substantial Variation Likely Driven by Neutral Processes, Genome Biol. Evol. 9 (2017) 1499-1512. https://doi.org/10.1093/gbe/evx097.

[15] M. Petersen, D. Armisén, R.A. Gibbs, L. Hering, A. Khila, G. Mayer, S. Richards, O. Niehuis, B. Misof, Diversity and evolution of the transposable element repertoire in arthropods with particular reference to insects, BMC Evol. Biol. 19 (2019) 11. https://doi.org/10.1186/s12862-018-1324-9. 
[16] M. Wu, J.L. Kostyun, L.C. Moyle, Genome Sequence of Jaltomata Addresses Rapid Reproductive Trait Evolution and Enhances Comparative Genomics in the Hyper-Diverse Solanaceae, Genome Biol. Evol. 11 (2019) 335-349. https://doi.org/10.1093/gbe/evy274.

[17] C. Vitte, O. Panaud, H. Quesneville, LTR retrotransposons in rice (Oryza sativa, L.): recent burst amplifications followed by rapid DNA loss, BMC Genomics. 8 (2007) 218. https://doi.org/10.1186/1471-2164-8-218.

[18] J.S. Hawkins, S.R. Proulx, R.A. Rapp, J.F. Wendel, Rapid DNA loss as a counterbalance to genome expansion through retrotransposon proliferation in plants, Proc. Natl. Acad. Sci. 106 (2009) 17811-17816. https://doi.org/10.1073/pnas.0904339106.

[19] Z.-Q. Zhang, Animal biodiversity: An outline of higher-level classification and survey of taxonomic richness (Addenda 2013), Zootaxa. 3703 (2013) 1-82.

[20] G. Giribet, G.D. Edgecombe, Reevaluating the Arthropod Tree of Life, Annu. Rev. Entomol. 57 (2012) 167-186. https://doi.org/10.1146/annurev-ento-120710-100659.

[21] M.A. Chebbi, T. Becking, B. Moumen, I. Giraud, C. Gilbert, J. Peccoud, R. Cordaux, The Genome of Armadillidium vulgare (Crustacea, Isopoda) Provides Insights into Sex Chromosome Evolution in the Context of Cytoplasmic Sex Determination, Mol. Biol. Evol. 36 (2019) 727-741. https://doi.org/10.1093/molbev/msz010.

[22] T. Becking, M.A. Chebbi, I. Giraud, B. Moumen, T. Laverré, Y. Caubet, J. Peccoud, C. Gilbert, R. Cordaux, Sex chromosomes control vertical transmission of feminizing Wolbachia symbionts in an isopod, PLOS Biol. 17 (2019) e3000438. https://doi.org/10.1371/journal.pbio.3000438.

[23] T. Becking, I. Giraud, M. Raimond, B. Moumen, C. Chandler, R. Cordaux, C. Gilbert, Diversity and evolution of sex determination systems in terrestrial isopods, Sci. Rep. 7 (2017) 1084. https://doi.org/10.1038/s41598-017-01195-4.

[24] F.A. Simão, R.M. Waterhouse, P. Ioannidis, E.V. Kriventseva, E.M. Zdobnov, BUSCO: assessing genome assembly and annotation completeness with single-copy orthologs, Bioinformatics. 31 (2015) 3210-3212. https://doi.org/10.1093/bioinformatics/btv351.

[25] L. Li, C.J. Stoeckert, D.S. Roos, OrthoMCL: identification of ortholog groups for eukaryotic genomes, Genome Res. 13 (2003) 2178-2189. https://doi.org/10.1101/gr.1224503.

[26] B. Valdebenito-Maturana, G. Riadi, TEcandidates: prediction of genomic origin of expressed transposable elements using RNA-seq data, Bioinformatics. 34 (2018) 39153916. https://doi.org/10.1093/bioinformatics/bty423.

[27] B.J. Haas, A. Papanicolaou, M. Yassour, M. Grabherr, P.D. Blood, J. Bowden, M.B. Couger, D. Eccles, B. Li, M. Lieber, M.D. Macmanes, M. Ott, J. Orvis, N. Pochet, F. Strozzi, N. Weeks, R. Westerman, T. William, C.N. Dewey, R. Henschel, R.D. Leduc, N. Friedman, A. Regev, De novo transcript sequence reconstruction from RNA-seq using the Trinity platform for reference generation and analysis, Nat. Protoc. 8 (2013) 14941512. https://doi.org/10.1038/nprot.2013.084.

[28] A.R. Quinlan, I.M. Hall, BEDTools: a flexible suite of utilities for comparing genomic features, Bioinformatics. 26 (2010) 841-842. https://doi.org/10.1093/bioinformatics/btq033.

[29] J. Romiguier, P. Gayral, M. Ballenghien, A. Bernard, V. Cahais, A. Chenuil, Y. Chiari, R. Dernat, L. Duret, N. Faivre, E. Loire, J.M. Lourenco, B. Nabholz, C. Roux, G. Tsagkogeorga, A. a.-T. Weber, L.A. Weinert, K. Belkhir, N. Bierne, S. Glémin, N. Galtier, 
Comparative population genomics in animals uncovers the determinants of genetic diversity, Nature. 515 (2014) 261-263. https://doi.org/10.1038/nature13685.

[30] B. Langmead, S.L. Salzberg, Fast gapped-read alignment with Bowtie 2, Nat. Methods. 9 (2012) 357-359. https://doi.org/10.1038/nmeth.1923.

[31] T. Carver, S.R. Harris, M. Berriman, J. Parkhill, J.A. McQuillan, Artemis: an integrated platform for visualization and analysis of high-throughput sequence-based experimental data, Bioinformatics. 28 (2012) 464-469. https://doi.org/10.1093/bioinformatics/btr703.

[32] J.S. Taylor, Y. Van de Peer, A. Meyer, Genome duplication, divergent resolution and speciation, Trends Genet. TIG. 17 (2001) 299-301.

[33] E.B. Holub, The arms race is ancient history in Arabidopsis, the wildflower, Nat. Rev. Genet. 2 (2001) 516-527. https://doi.org/10.1038/35080508.

[34] O. Lespinet, Y.I. Wolf, E.V. Koonin, L. Aravind, The role of lineage-specific gene family expansion in the evolution of eukaryotes, Genome Res. 12 (2002) 1048-1059. https://doi.org/10.1101/gr.174302.

[35] S. Kim, M.-J. Yoo, V.A. Albert, J.S. Farris, P.S. Soltis, D.E. Soltis, Phylogeny and diversification of B-function MADS-box genes in angiosperms: evolutionary and functional implications of a 260-million-year-old duplication, Am. J. Bot. 91 (2004) 2102-2118. https://doi.org/10.3732/ajb.91.12.2102.

[36] L. Carretero-Paulet, M.A. Fares, Evolutionary dynamics and functional specialization of plant paralogs formed by whole and small-scale genome duplications, Mol. Biol. Evol. 29 (2012) 3541-3551. https://doi.org/10.1093/molbev/mss162.

[37] S.P. Otto, J. Whitton, Polyploid incidence and evolution, Annu. Rev. Genet. 34 (2000) 401-437. https://doi.org/10.1146/annurev.genet.34.1.401.

[38] S. Hoegg, H. Brinkmann, J.S. Taylor, A. Meyer, Phylogenetic timing of the fish-specific genome duplication correlates with the diversification of teleost fish, J. Mol. Evol. 59 (2004) 190-203. https://doi.org/10.1007/s00239-004-2613-z.

[39] S. Magadum, U. Banerjee, P. Murugan, D. Gangapur, R. Ravikesavan, Gene duplication as a major force in evolution, J. Genet. 92 (2013) 155-161.

[40] T.C. Mathers, Y. Chen, G. Kaithakottil, F. Legeai, S.T. Mugford, P. Baa-Puyoulet, A. Bretaudeau, B. Clavijo, S. Colella, O. Collin, T. Dalmay, T. Derrien, H. Feng, T. Gabaldón, A. Jordan, I. Julca, G.J. Kettles, K. Kowitwanich, D. Lavenier, P. Lenzi, S. Lopez-Gomollon, D. Loska, D. Mapleson, F. Maumus, S. Moxon, D.R.G. Price, A. Sugio, M. van Munster, M. Uzest, D. Waite, G. Jander, D. Tagu, A.C.C. Wilson, C. van Oosterhout, D. Swarbreck, S.A. Hogenhout, Rapid transcriptional plasticity of duplicated gene clusters enables a clonally reproducing aphid to colonise diverse plant species, Genome Biol. 18 (2017). https://doi.org/10.1186/s13059-016-1145-3.

[41] A. Vandel, Faune de France. Isopodes Terrestres (Deuxième Partie), Lechevalier, Lechevalier, Paris, 1962.

[42] S. Aparicio, J. Chapman, E. Stupka, N. Putnam, J. Chia, P. Dehal, A. Christoffels, S. Rash, S. Hoon, A. Smit, M.D.S. Gelpke, J. Roach, T. Oh, I.Y. Ho, M. Wong, C. Detter, F. Verhoef, P. Predki, A. Tay, S. Lucas, P. Richardson, S.F. Smith, M.S. Clark, Y.J.K. Edwards, N. Doggett, A. Zharkikh, S.V. Tavtigian, D. Pruss, M. Barnstead, C. Evans, H. Baden, J. Powell, G. Glusman, L. Rowen, L. Hood, Y.H. Tan, G. Elgar, T. Hawkins, B. Venkatesh, D. Rokhsar, S. Brenner, Whole-Genome Shotgun Assembly and Analysis of the Genome of Fugu rubripes, Science. 297 (2002) 1301-1310. https://doi.org/10.1126/science.1072104. 
[43] E. Blass, M. Bell, S. Boissinot, Accumulation and Rapid Decay of Non-LTR Retrotransposons in the Genome of the Three-Spine Stickleback, Genome Biol. Evol. 4 (2012) 687-702. https://doi.org/10.1093/gbe/evs044.

[44] M. Naville, S. Henriet, I. Warren, S. Sumic, M. Reeve, J.-N. Volff, D. Chourrout, Massive Changes of Genome Size Driven by Expansions of Non-autonomous Transposable Elements, Curr. Biol. 29 (2019) 1161-1168.e6. https://doi.org/10.1016/j.cub.2019.01.080.

[45] C.M. Vicient, A. Suoniemi, K. Anamthawat-Jónsson, J. Tanskanen, A. Beharav, E. Nevo, A.H. Schulman, Retrotransposon BARE-1 and Its Role in Genome Evolution in the Genus Hordeum, Plant Cell. 11 (1999) 1769-1784. https://doi.org/10.1105/tpc.11.9.1769.

[46] J.L. Bennetzen, J. Ma, K.M. Devos, Mechanisms of Recent Genome Size Variation in Flowering Plants, Ann. Bot. 95 (2005) 127-132. https://doi.org/10.1093/aob/mci008.

[47] B. Nystedt, N.R. Street, A. Wetterbom, A. Zuccolo, Y.-C. Lin, D.G. Scofield, F. Vezzi, N. Delhomme, S. Giacomello, A. Alexeyenko, R. Vicedomini, K. Sahlin, E. Sherwood, M. Elfstrand, L. Gramzow, K. Holmberg, J. Hällman, O. Keech, L. Klasson, M. Koriabine, M. Kucukoglu, M. Käller, J. Luthman, F. Lysholm, T. Niittylä, Å. Olson, N. Rilakovic, C. Ritland, J.A. Rosselló, J. Sena, T. Svensson, C. Talavera-López, G. Theißen, H. Tuominen, K. Vanneste, Z.-Q. Wu, B. Zhang, P. Zerbe, L. Arvestad, R. Bhalerao, J. BohImann, J. Bousquet, R.G. Gil, T.R. Hvidsten, P. de Jong, J. MacKay, M. Morgante, K. Ritland, B. Sundberg, S.L. Thompson, Y.V. de Peer, B. Andersson, O. Nilsson, P.K. Ingvarsson, J. Lundeberg, S. Jansson, The Norway spruce genome sequence and conifer genome evolution, Nature. 497 (2013) 579-584. https://doi.org/10.1038/nature12211.

[48] M. Dupeyron, S. Leclercq, N. Cerveau, D. Bouchon, C. Gilbert, Horizontal transfer of transposons between and within crustaceans and insects, Mobile DNA. 5 (2014) 4. 
421 Table 1. Comparison of gene content in the Armadillidium vulgare and Armadillidium

422 nasatum genomes.

423

\begin{tabular}{|c|c|c|c|c|}
\hline & Genes & $\begin{array}{l}\text { Genes common } \\
\text { to both species }\end{array}$ & $\begin{array}{l}\text { Species-specific } \\
\text { genes }^{\text {b }}\end{array}$ & $\begin{array}{l}\text { Single-copy species- } \\
\text { specific genes }\end{array}$ \\
\hline \multicolumn{5}{|l|}{ Armadillidium vulgare } \\
\hline Total nb proteins & 19051 & 10815 & 1898 & 6338 \\
\hline $\mathrm{Nb}$ proteins of unknown function & 8588 & 1388 & 871 & 2133 \\
\hline$\%$ proteins of unknown function & $45.08 \%$ & $12.83 \%$ & $45.89 \%$ & $33.65 \%$ \\
\hline \multicolumn{5}{|l|}{ Armadillidium nasatum } \\
\hline Total nb proteins & 14636 & 9429 & 1037 & 4170 \\
\hline $\mathrm{Nb}$ proteins of unknown function & 5353 & 2645 & 612 & 2397 \\
\hline$\%$ proteins of unknown function & $36.57 \%$ & $28.05 \%$ & $59.02 \%$ & $57.48 \%$ \\
\hline
\end{tabular}

424

$425{ }^{\text {a }}$ clustered in 8,036 orthogroups

$426{ }^{b}$ clustered in 1,104 orthogroups

427 
428 Table 2. Comparison of repeat content in the Armadillidium vulgare and Armadillidium 429 nasatum genomes.

430

\begin{tabular}{lllllll}
\hline & \multicolumn{7}{c}{ Armadillidium vulgare (a) } & Armadillidium nasatum (b) & Genome size difference (a-b) \\
\cline { 2 - 7 } & Count & $\begin{array}{l}\text { Cumulative } \\
\text { length (bp) }\end{array}$ & Count & $\begin{array}{l}\text { Cumulative } \\
\text { length (bp) }\end{array}$ & $\begin{array}{l}\text { Cumulative } \\
\text { length (bp) }\end{array}$ & $\begin{array}{l}\text { \% cumulative } \\
\text { length }\end{array}$ \\
\hline Genome & $\mathrm{n} / \mathrm{a}$ & 1725108002 & $\mathrm{n} / \mathrm{a}$ & 1223175971 & 501932031 & $\mathrm{n} / \mathrm{a}$ \\
All TEs & 2132032 & 854113876 & 1544121 & 484727234 & 369386642 & $73.59 \%$ \\
DNA transposons & 986943 & 279615254 & 750157 & 157019308 & 122595946 & $24.42 \%$ \\
LINEs & 796433 & 417726622 & 532873 & 247894416 & 169832206 & $33.84 \%$ \\
SINEs & 37622 & 5933232 & 32834 & 4978843 & 954389 & $0.19 \%$ \\
LTRs & 246423 & 135610359 & 179870 & 63190568 & 72419791 & $14.43 \%$ \\
Retroposons & 64611 & 15228409 & 48387 & 11644099 & 3584310 & $0.71 \%$ \\
& & & & & & \\
Tandem repeats & 2536308 & 303620084 & 2047530 & 285430073 & 18190011 & $3.62 \%$ \\
Other repeats & 227683 & 17582771 & 217427 & 20436134 & -2853363 & $-0.57 \%$ \\
\hline
\end{tabular}

431

432 
Table 3. Comparison of expressed copies of transposable elements in the Armadillidium

434 vulgare and Armadillidium nasatum genomes.

435

\begin{tabular}{lllll}
\hline & \multicolumn{2}{l}{ Armadilidium vulgare } & \multicolumn{2}{l}{ Armadillidium nasatum } \\
\cline { 2 - 5 } & Count & $\%$ & Count & $\%$ \\
\hline LINEs & 2822 & $53.08 \%$ & 657 & $52.18 \%$ \\
SINEs & 6 & $0.11 \%$ & 0 & $0 \%$ \\
LTRs & 779 & $14.65 \%$ & 258 & $20.49 \%$ \\
DNA transposons & 1374 & $25.84 \%$ & 318 & $25.26 \%$ \\
Retroposons & 314 & $5.91 \%$ & 11 & $0.87 \%$ \\
Others & 22 & $0.41 \%$ & 15 & $1.19 \%$ \\
All transposable elements & 5317 & $100 \%$ & 1259 & $100 \%$ \\
\hline
\end{tabular}

436

437 
439 vulgare (red) and Armadillidium nasatum (blue). Proteins common to both genomes $(9,429$

440 for $A$. nasatum and 10,815 for $A$. vulgare) correspond to 8,036 orthogroups.

441

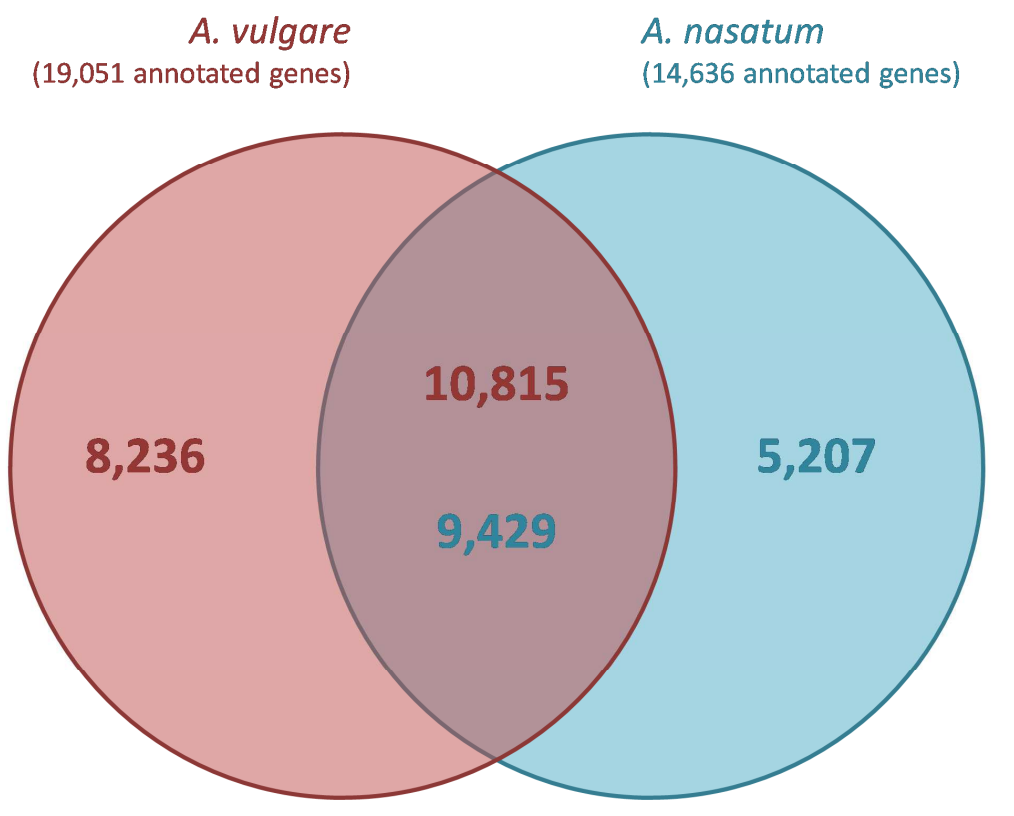

442 
444 Figure 2. Content of the Armadillidium vulgare (A) and Armadillidium nasatum (B) genomes.

445 (C) Comparison of the cumulative length (in megabases) of various genomic categories in $A$. $446 \quad$ vulgare and A. nasatum.

447

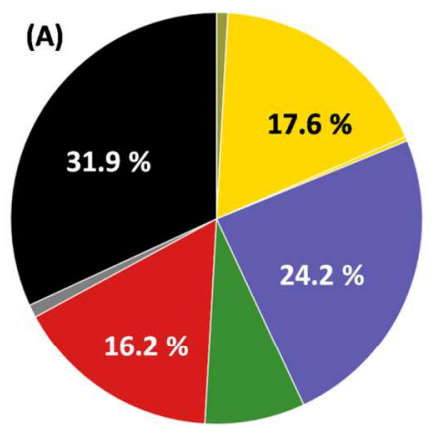

(C)

(B)

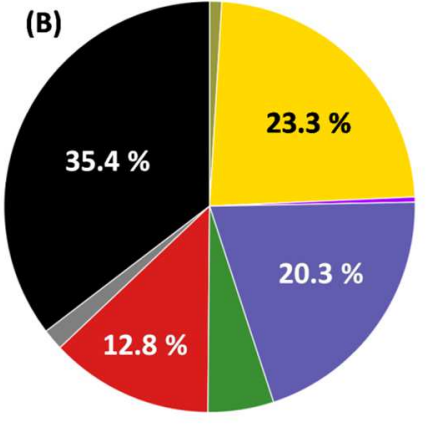

A. vulgare

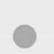

(1)

3.6

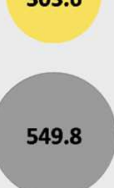

135.6

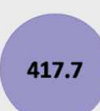

417.7

279.6
A. nasatum

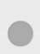

0

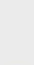

○

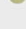

285.4

432.6

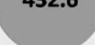

63.2

247.9

157 
450 Figure 3. Frequency distribution of transposable element families according to the

451 divergence of individual copies to their respective family consensus sequences. Transposable

452 elements identified in Armadillidium vulgare are shown in the upper part of the figure and

453 those identified in Armadillidium nasatum in the lower part. The red arrow indicates the

454 estimated genetic distance between $A$. vulgare and $A$. nasatum.

455
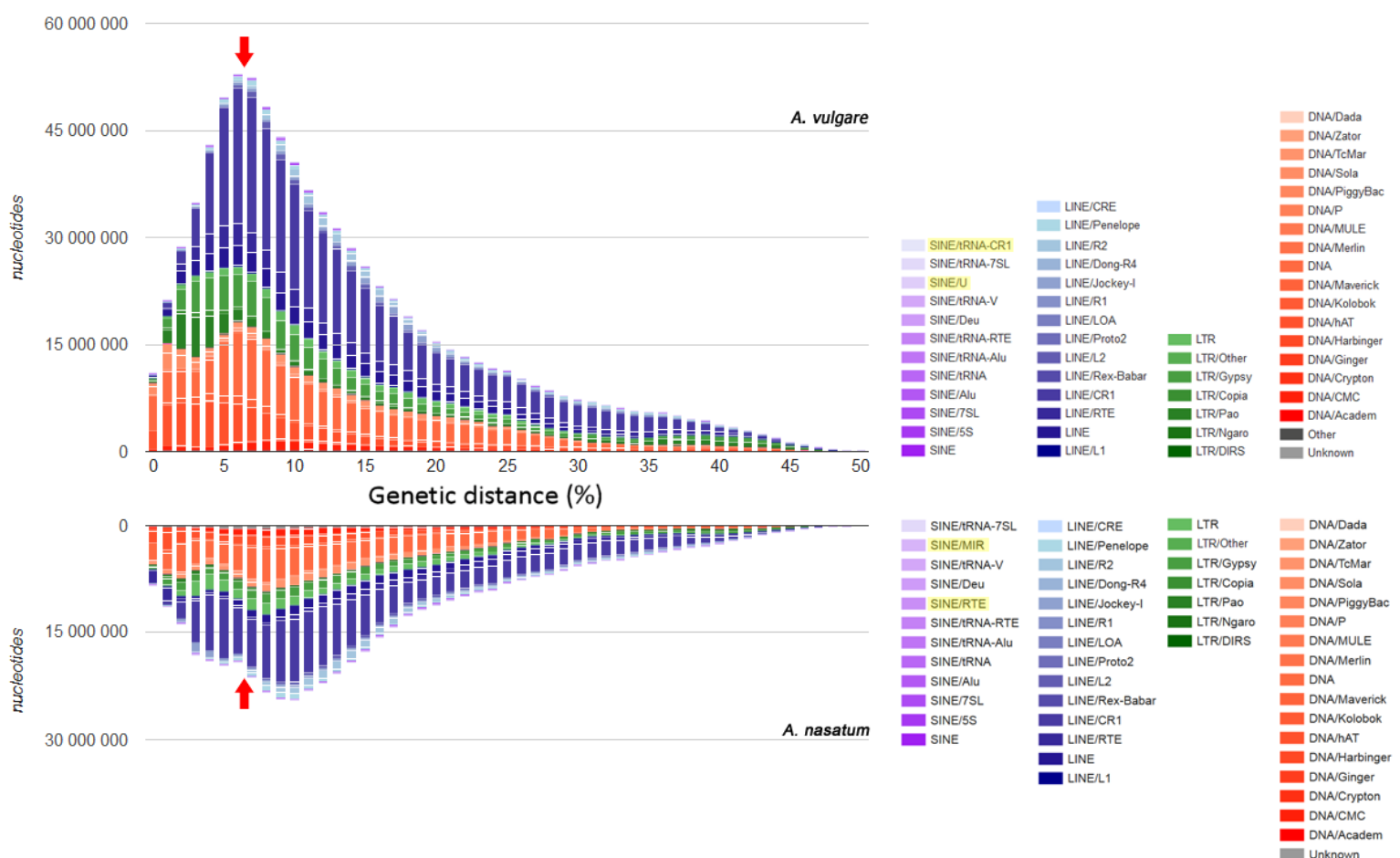
459 Figure 4. Comparison of the most expressed transposable element families in the

460 Armadillidium vulgare (top) and Armadillidium nasatum (bottom) genomes. The cumulative

461 expression (in reads per kilobase of exon model per million mapped) of the 15 most expressed families are shown. Error bars indicate standard deviations.

463

464

465

466
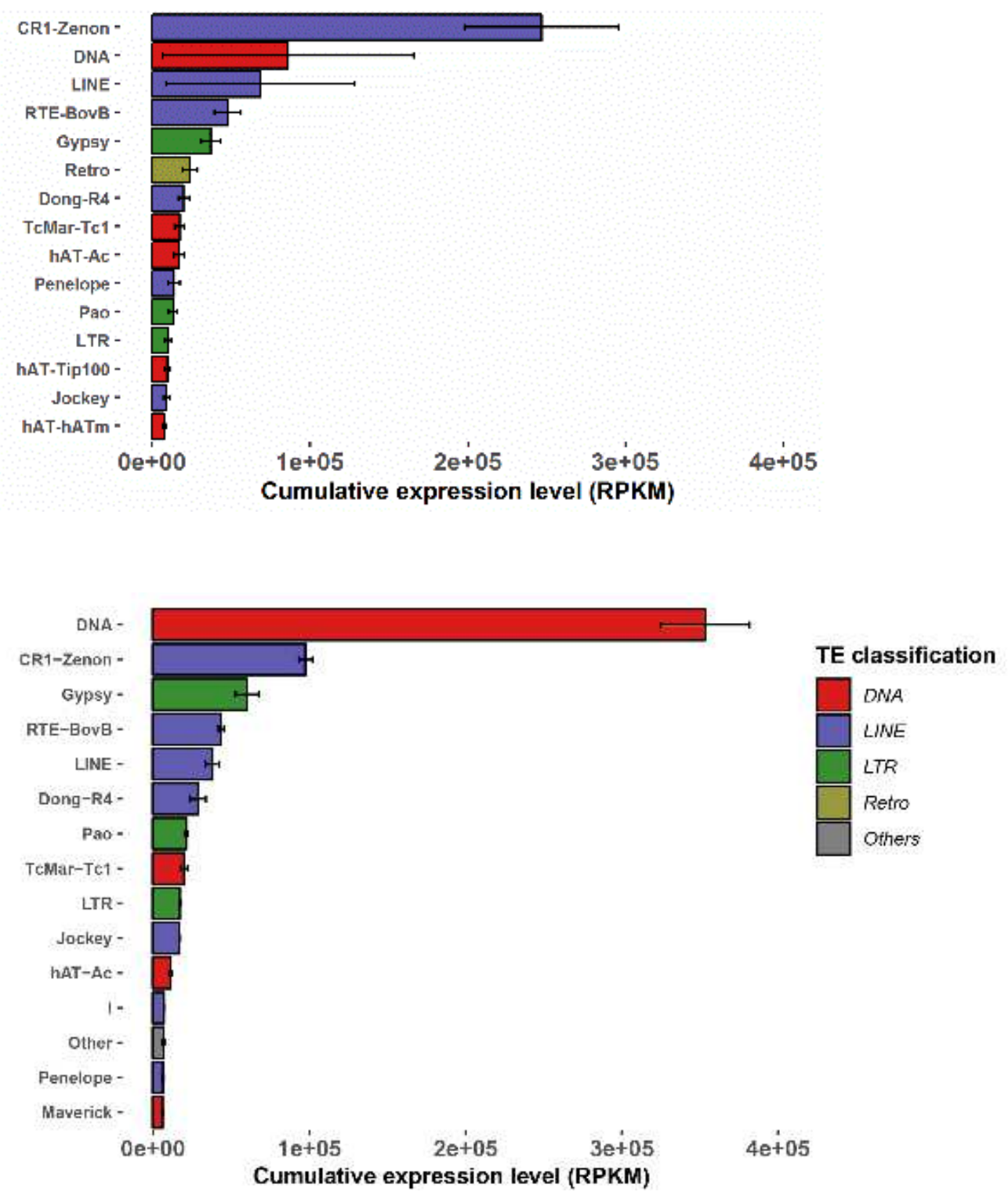